\title{
Results from the third flight of ANITA
}

\author{
Abigail Vieregg ${ }^{1, \star}$ for the ANITA Collaboration \\ ${ }^{1}$ Dept. of Physics, Enrico Fermi Institute, Kavli Institute for Cosmological Physics, University of Chicago
}

\begin{abstract}
We summarize results from the third flight of the Antarctic Impulsive Transient Antenna (ANITA), a NASA long-duration balloon payload that searches for radio emission from the interactions of ultra-high-energy neutrinos and cosmic rays. ANITAIII was launched in December 2014 and flew for 22 days. We report the results from multiple analyses of the data, which search for Askaryan radio emission from neutrinos interacting in the Antarctic ice as well as geomagnetic radio emission from extensive air showers (EASs) induced by cosmic rays or a tau lepton created in an in-earth tau neutrino interaction. In the most sensitive Askaryan neutrino search, we find one event on a pre-unblinding background of $0.7_{-0.3}^{+0.5}$. Across all searches, including a dedicated EAS search, we find a total of 28 EAS-like events. One of these events is consistent with an upward-traveling EAS, with a post-unblinding background estimate of $\lesssim 10^{-2}$.
\end{abstract}

The Antarctic Impulsive Transient Antenna (ANITA), a NASA long-duration balloon payload, monitors the Antarctic ice sheet for impulsive, broadband radio emission (180 - $1200 \mathrm{MHz}) \mathrm{caused}$ by high-energy astrophysical and cosmogenic neutrino and cosmic-ray interactions. ANITA is sensitive to two types of impulsive particle-induced radio signals. The first is Askaryan emission from neutrino-induced cascades in the Antarctic ice, which appears as a vertically-polarized signal at the ANITA payload due to geometric effects combined with the radial polarization of the Cherenkov cone and Fresnel effects at the surface. The second is geomagnetic emission from extensive air showers (EAS) induced either by cosmic rays or by decaying tau leptons (daughter particles from tau neutrino interactions in the Earth). Emission from EASs appears mostly horizontally polarized, since ANITA flies in Antarctica, where the Earth's magnetic field is mainly vertical.

The third flight of ANITA (ANITA-III) was launched on 18 December 2014 and flew for 22 days. The primary upgrades from ANITA-II are the addition of 8 more antennas, the implementation of a new impulsive full-band-only trigger, and the use of new lower-noise radio-frequency amplifiers. For more details see [1]. We have performed four separate analyses of data from ANITA-III, each searching for Askaryan neutrino candidate events and/or EAS candidate events. The results are discussed fully in [1] and [2], and we summarize them here.

Polarization information about each event provides a straightforward way to separate the two search regions. Three of the searches were optimized for vertically-polarized Askaryan neutrino signals, but maintained sensitivity to the horizontally-polarized EAS signal region. The fourth search was a dedicated EAS search, which achieved a lower expected background in the signal region through implementation of signal-shape criteria specific to EAS events.

All analyses of ANITA data must battle two main sources of background: thermal-noise-like events and events due to anthropogenic noise. Thermal-noise-like events are relatively straightforward

^e-mail: avieregg@kicp.uchicago.edu 

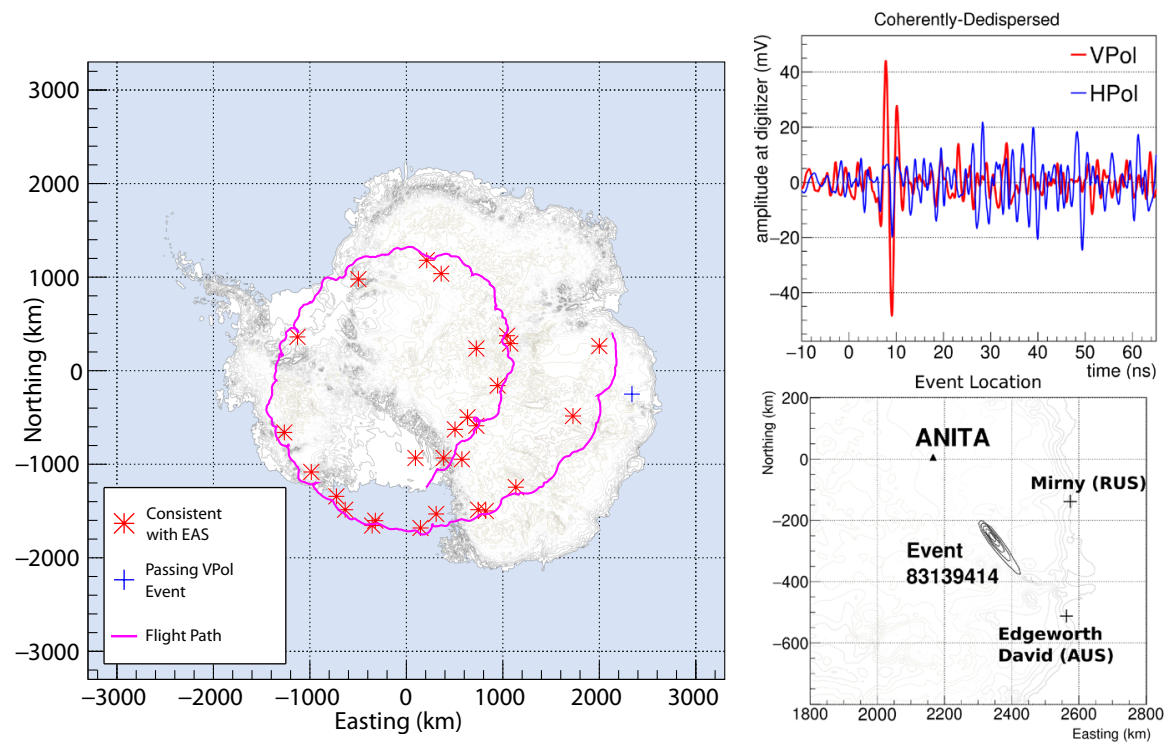

Figure 1. Left: The 26 EAS-like events that come from below the horizon found by any analysis (red), and the single remaining event in the most sensitive of the three Askaryan neutrino searches (blue). Upper Right: The coherently summed and dedispersed waveform for the single remaining event in the Askaryan neutrino signal region. Lower Right: The location (with uncertainties) of the event projected onto the continent, as well as the location of ANITA at the time of the event, and the closest known locations of human activity shown. From Reference [1].

to deal with: all analyses use either a sideband region or a decimated data set to design cuts to separate signal-like events from thermal-noise-like backgrounds. Separating events due to anthropogenic noise with high fidelity relies on geographic clustering or geographic binning.

We then estimate the background in the signal region and the analysis efficiency on Monte Carlo neutrino events as well as events from calibration sources. Since we have observed EASs in previous flights of ANITA [3], we can use cuts that depend on the known signal properties of EAS events to maintain a high analysis efficiency and achieve a lower background in the EAS search. In the dedicated EAS search, additional signal-shape cuts (e.g., cross correlating events with an EAS template) were applied to reduce the background. In the Askaryan neutrino searches, we are more agnostic toward signal shape, only requiring that the signal be impulsive.

The most sensitive Askaryan neutrino search (defined using the best expected limit) found one event in the signal region on a background estimate of $0.7_{-0.3}^{+0.5}$. We also found a total of 28 EAS candidate events across all searches, including the dedicated EAS template-matching search. Two of these events originate from below the horizontal $\left(0^{\circ}\right.$ in elevation) but above $-6^{\circ}$ in elevation (which is the horizon as viewed by ANITA) and are consistent with cosmic-ray EAS events where the radio emission does not reflect off of the surface of Antarctica. The remaining 26 originate from below the horizon. The left panel of Figure 1 shows the candidate EAS events that originate from below the horizon and the single remaining event in the Askaryan signal region. The right panel shows the waveforms for the single event in the Askaryan signal region, and a projection onto the continent. We proceed to set a limit on the diffuse flux of ultra-high-energy neutrinos, which is shown in the left panel of Figure 2. 

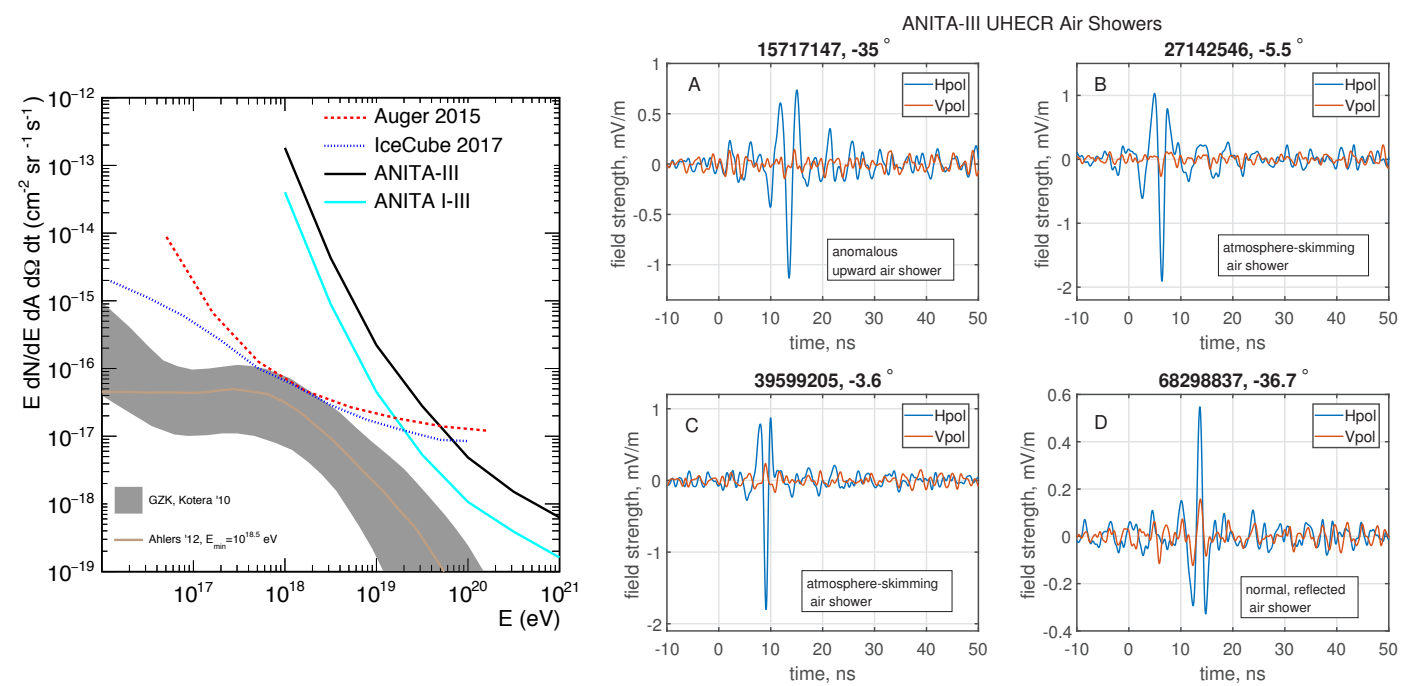

Figure 2. Left: The ANITA-III limit on the all-flavor diffuse ultra-high-energy neutrino flux and a combined limit from ANITA-I-III, using the ANITA-III limit shown here and the published ANITA-I and ANITA-II limits [5, 6]. Ultra-high-energy neutrino limits from Auger [7] and IceCube [8], as well as two cosmogenic neutrino models [9, 10] are also shown. From Reference [1]. Right: The non-inverted, upward-going EAS from below the horizon (Panel A), two non-inverted cosmic-ray EAS events from above the horizon (Panels B and C), and and an example inverted event from a cosmic-ray EAS where the radio emission reflected off of the surface of Antarctica (Panel D). From Reference [2].

Of the 26 EAS candidate events that originate from below the horizon, 25 have a polarity that is consistent with radio emission reflecting off of the surface of Antarctica from a downward-going cosmic ray. One below-horizon event, shown in panel A of the right side of Figure 2 has a polarity consistent with no reflection (see e.g., panels $\mathrm{B}$ and $\mathrm{C}$ of the right side of Figure 2). The background estimate is $\lesssim 10^{-2}$ for this event. This event is also consistent with a similar event observed in ANITA-I [4], with a similarly low background estimate. In the hypothesis of an EAS from the decay of a tau lepton created in a tau neutrino interaction, the steep upgoing angle of this event creates tension with the standard model neutrino cross section.

\section{References}

[1] P. Gorham et al. PRD 98:022001 (2018)

[2] P. Gorham et al. Accepted at PRL, arXiv:1803:05088 (2018)

[3] H. Schoorlemmer et al. Ast. Phys. 77:32-43 (2016); S. Hoover et al. PRL 105:151101 (2010)

[4] P. Gorham et al. PRL 117:071101 (2016)

[5] P. Gorham et al. PRL 103:051103 (2009)

[6] P. Gorham et al. PRD 82:022004 (2010); P. Gorham et al. PRD 85:049901 (2011)

[7] A. Aab et al. PRD 91:092008 (2015)

[8] M. Aartsen et al. PRL 119:259902 (2017)

[9] K. Kotera et al. JCAP 10:013 (2010)

[10] M. Ahlers et al. PRD 86:083010 (2012) 